\title{
User Recommendation in OSN
}

\author{
ChristiyanaArulselvi $\mathrm{A}^{\mathrm{a}, 1}$, SendhilkumarS ${ }^{\mathrm{b}}$ and Mahalakshmi $\mathrm{G} \mathrm{S}^{\mathrm{c}}$ \\ a Assistant Professor, Dept of Computer Applications, SRM Institute of Science \& \\ Technology, Chennai, India \\ ${ }^{b}$ Associate Professor, Dept of IT' Anna University, Chennai, India \\ ${ }^{c}$ Associate Professor, Dept of CSE, Anna University, Chennai, India
}

\begin{abstract}
Web based Social Network (SN) is one of the popular mediums where the information is shared among users. The combined data from different sources available on the open web needs to be evaluated for its quality and trustworthiness. A trustable community can be formed, if the social networking applications, media, forums, etc., can automatically judge the user who requests to join the community. It is necessary to know the trustability of the persons within the community to share the official and personal information. Therefore, a trust model has become an essential part of Online Social Network (OSN) to find the trustworthiness of the requester. Significance of the work detailed here is to model a trustworthy user suggestion in OSN applications.
\end{abstract}

Keywords. User recommendation, collaborative filtering, interaction data, Trust, OSN

\section{Introduction}

The momentous development in the technological innovation incredibly affects medium in which people communicate with each other. The impact is evidenced with the way, web-based media for example broadcast communication framework, texting, WWW and interpersonal interaction sites are working. Social networks allow users to share the events happening on the open forum through posting photos, messages and status updates. It helps the users to share a wide range of data among friends. Despite the fact that OSNs permit to associate with companions, Web 2.0 and OSNs encourage the way for bogus and misdirecting data which needs check mechanism [1].OSNs today demands people in decision making. Current social networks being an online ommunity requires recommendation from third party for decision making. The recommendation in OSN could beuser -itemsrecommendation and user-user recommendation. In user based community users are prone to have the thread enforced by the imposters in order to abuse the users Anticipating and eradicating such imposters is the requisite for the virtual environments like OSN[2]. In addition to the eradication of the imposters, suggesting trustworthy user will protect and enhance the wellbeing of the communityIn user based community users are prone to have the threat enforced by the imposters in order to abuse the users.Anticipating and eradicating such imposters is the requisite for the virtual environments like OSN[2]. 


\section{Related Works}

A recommendation system can be described as a decision-making method that allows the user to make the choice [3]. The various recommendation systems that had been proposed in the literature [4] to solve the shortcomings in OSN are collaborative filtering, content-based filtering, demographic, knowledge-based [5, 6, 7], utility-based recommendation and hybrid recommendation [8]. Among these recommendation systems, the proposed system adopts collaborative filtering recommendation system. Generally, item recommendation in OSN is achieved from the user's reviews on the item or the user's past online purchase behaviour. User recommendation could be achieved through the user profile [9] which contains users' demographic information like language, locality, interest, etc., and users' interaction information. The profile information is the factors that influence the user's personalized recommendation. Attitude, behavior and experience are the three main sources of trust computation [10]. Interaction behavior of the user reflects the real closeness between or among users. Krishnaprasad Thirunarayan \& Pramod Anantharam[11] analyzed friends' conversational information in Facebook to determine trust between friends in social networks. Sibel Adali et al. [12] mentioned trust is an important but complex among the users of OSN and proposed a trust model based on the user behaviour in terms of conversational and propagational data available in OSN for twitter data. Sapna Gambhir \&Vinod Kumar [13] proposed a trust computation system in OSN between users based on time duration of various actions (message, comments, shares, likes, tags) that was happened.

\section{Collaborative Filtering Based Trust Model}

OSN in the present state of affairs is a major instrument for social network. In such virtual environment, people are looking for genuine users to make the interactions healthier and interesting. A recommendation system is a form of decision support system that is used to ascertain user's favourites. Users get suggestions from the system based on their interests and those of other users. Recommendation systems is an important tool that analyzes users' actions and make recommendations based on their favorites.The aim of the proposed personalized recommendations is to 1) find trustworthy users who can help OSN users, 2) screen friend requests by detecting imposters (untrustworthy users), and 3) maintain a secure and healthy group.

Though the collaborative filtering technique is mostly used to recommend items based on similarity among users, the proposed system recommend a user (alter) to another user (alter) based on the reputation gained by the prime user on alter based on interaction. The proposed research work uses frequency pattern among various type of user behavior patterns described by Park DHet al. [16]. Trust value (user rating) could be predicted using stochastic model once the latent features are identified [15][18]. The process of trust prediction as proposed by Christiyana et al [2] and user recommendation for the proposed work is explained below:

1) Compute the prime user's reputation on another user $\mathrm{x}$ based on prime user's interaction using stochastic differential equation.

2) Compute trust from the reputation value, which defines the closeness of prime user with user $\mathrm{x}$. 
3) Recommend user $x$ to ( $n-x)$ users in prime user's community or to give suggestion to the users who received the friend request from user $\mathrm{x}$. This process of recommendation is explained herewith.

\section{User Recommendation in OSN}

In OSN environs, the recommendation framework is utilized to supply prospective users to the focused based on the user actions. Though the individuals are overwhelmed with data detecting an individual who is likely to be included in the companion list isn't an issue any longer but identifyingand offering the prospective users to the focused may be a crucial assignment in OSN. Aguideline process was designed to bridge the fissure among collecting, investigating, cleaning the accessible data and presenting it to the target audience. Hence, the proposed strategy employs reputation-based user believe framework to identify and recommend the prospective user to the requested. Sample trust value obtained using stochastic model and Bayesian classification process by Christiyana et al [2] isgiven in Table 1.

Table 1.Trustability of ego user (p) on every other users (n-p) of ego user community

\begin{tabular}{lllcc}
\hline User id & Like & Comment & Chat & Class \\
\hline u1 & 0.05 & 0.07 & 0.30 & $\mathrm{R}$ \\
u2 & 0.04 & 0.12 & 0.01 & $\mathrm{R}$ \\
u3 & 0.06 & 0.00 & 0.32 & $\mathrm{R}$ \\
u4 & 0.05 & 0.03 & 0.37 & $\mathrm{R}$ \\
u5 & 0.05 & 0.13 & 0.04 & $\mathrm{R}$ \\
u6 & 0.06 & 0.15 & 0.01 & $\mathrm{R}$ \\
\hline
\end{tabular}

The fields Like, Comment and Chat in Table 1 are the parameters used for the current research work to compute the trustability of the ego user on every other user in the community. These parameters are extracted from individual user's timeline and chat sessions in FaceBook. Values of these parameters are computed using stochastic model proposed by Christiyana et al [2]. The field named 'Class' in Table 1 defined the accepted (A) and refused (R) labels as trusted and untrusted users respectively. The corresponding output were classified using Bayesian classier with $96.7 \%$ accuracy. The precision test of the classifier showed that 96.7 percent of trusted users classified are reliable [2].The recommendation system thus built using stochastic and Bayesian classification is evaluated for its effectiveness as in section 5 . 


\section{Performance of User Recommendation}

To assess the performance of the current research work defined for the trustworthy user community, the groups generated by the current research work must be analyzed. The trusted and untrusted user groups formed by the current research work is assessed using the statistical measures called Average Root Mean Square Error (ARMSE).Expected groups' consistency is measured using Average Root Mean Square Error (ARMSE), a statistical metric. The sample set of ego users wereprovided with the expected trust groups (Trusted and Untrusted users) created by the current system and their feedbacks were measured using the Average Root Mean Square Error (ARMSE) metric. The lower the error value, the higher the expected group's quality. Figure 1 shows the error value produced by ARMSE metric for the predicted trusted and untrusted group

\section{Average Root Mean}

Square Error

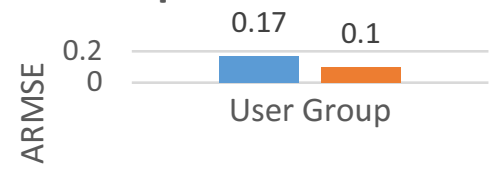

Trusted Users Untrusted Users

Figure 1. User recommendation - Performance evaluation

From the Fig. 1 it is observed that the average of the ARME computed for the trusted and untrusted groupswere 0.135 , which is approximately 0.14 . Therefore, $86 \%$ of the friend recommendation proposed by the current method is accurate in terms of ego user's confidence expectations.A recommendation system, which is a form of decision support system, assists the requested (targeted) in predicting the appropriate users in order to make a friend request decision. The proposed recommendation mechanism is based on the user's interaction experience, with frequency of interaction being used to calculate the user's confidence.

\section{Conclusion}

The work reported here provides a collaborative filtering approach for a user (friend) recommendation towards the friend request by considering various factors like frequency and content of interaction. A user recommendation for a friend request is a challenging task because the current user recommendation is based on the link structure between users. The proposed work overcomes the drawbacks of existing system by identifying the potential users with respect to the prime (ego) user and the mutual interest between the requester and the requested. The research reported here is to overcome the problems of friend request in OSN. A trusted user is recommended to alter of the ego user who is receiving friend request from another alter or to raise the friend request towards another trusted similar user. 


\section{Acknowledgements}

This Publication is an outcome of the R\&D work undertaken in the project under the Visvesvaraya PhD Scheme (Unique Awardee Number: VISPHD-MEITY-2959) of Ministry of Electronics \& Information Technology, Government of India, being implemented by Digital India Corporation (formerly Media Lab Asia).

\section{References}

[1] Sonja Grabner-Kräuter\&Sofie Bitter .Trust in online social networks: A multifaceted perspective. Forum for Social Economics, 2015, vol. 44. No. 1, pp.48-68, Routledge.

[2] ChristiyanaArulselvi A, Sendhilkumar S \&Mahalakshmi GS .Provenance based Trust computation for Recommendation in Social Network .In proceeding of International Conference on Informatics and Analytics (ICIA-16), (Pondicherry Engineering College, Pondicherry, India, 25th - 26th August 2016), ACM Article No. 114, DOI: 10.1145/2980258.2982105.

[3] ChristiyanaArulselvi. A, SendhilKumar S, and Mahalakshmi G S.Hybrid User Recommendation in Online Social Network.In Computational Vision and Bio Inspired Computing, 2018.pp. 134-151. Springer, Cham.

[4] SreeParvathy V \&Ratheesh TK .Friend recommendation system for online social networks: A survey. In proceedings of International conference of Electronics, Communication and Aerospace Technology, Coimbatore, India, 2017, pp. 359-365.

[5] Burke R Hybrid .Recommender systems: survey and experiments. User Model User-adapted Interact 2002. vol. 12. no. 4. pp. 331-170.

[6] Pathak D, Matharia S \& Murthy C .Nova: Hybrid book recommendation engine.In proceedings of 3rd International Advance Computing IEEE Conference, Ghaziabad, India, 2013, pp. 977-982.

[7] Adomavicius G \&Tuzhilin A 2005. Toward the next generation of recommender system: A survey of the State-of-the-Art and Possible Extensions. IEEE Transactions on Knowledge and Data Engineering, vol. 17, no. 6, pp. 734-49.

[8] Jannach D, Zanker M, Felfernig A \& Friedrich G, Recommender systems: An introduction, Cambridge University Press, 2010.

[9] Bobadilla J, Ortega F, Hernando A \& Gutierrez A .Recommender systems survey. KnowledgeBased Systems, 2013.vol. 46,pp. 109-132.

[10] WanitaSherchan, Surya Nepal \& Cecile Paris. A Survey of Trust in Social Networks. Journal of ACM Computing Surveys, 2013, vol. 45(4), Article no. 47. pp. 1-33.

[11] KrishnaprasadThirunarayan\&PramodAnantharam .Some Trust Issues in Social Networks and Sensor Networks. In proceedings of IEEE International Symposium on Collaborative Technologies and Systems, Chicago, IL, USA, 2010, pp. 573-580.

[12] SibelAdali, Robert Escriva, Mark K Goldberg, MykolaHayvanovych, Malik Magdon Ismail, Boleslaw K Szymanski, William A Wallace \& Gregory T Williams .Measuring Behavioral trust in Social Networks. In proceeding of IEEE International Conference on Intelligence and Security Informatics, Vancouver, BC, Canada. 2010.pp. 150-152.

[13] SapnaGambhir\&Vinod Kumar .Bidirectional Trust Calculation in Online Social Networks. In proceedings of 4th International Conference on Reliability. Infocom Technologies and Optimization, Noida, India. 2015. pp. 1-6.

[14] Isinkaye FO, Folajimi YO \&Ojokoh BA. Recommendation systems: Principles, methods and evaluation. Egyptian Informatics Journal, 2015. vol. 16, no. 3, pp. 261-273.

[15] Wei-Po Lee \&Chaun Yuan Ma.Enhancing Collaborative recommendation performance by combining user preference and Trust-Distrust propagation in Social Network. Knowledge-Based Systems, 2016. vol. 106, pp. 125-134.

[16] Park DH, Kim HK, Choi IY, Kim JK .A literature review and classification of recommender systems research. Expert Systems with Applications, 2012, vol. 39, no. 11, pp. 10059-10072.

[17] Christiyana Arulselvi A, Dhanalakshmi T \&Sendhilkumar S .Reputation based Trust Computation for Social Network.National Conference on Intelligent Computing and Data Analytics, Anna University, Chennai, 24th March 2016, pp. 183-193.

[18] AnkitKumar,Vijayakumar Varadarajan,C.Veluvolug. Black hole attack detection in vehicular ad-hoc network using secure AODV routing algorithm .Microprocessors and Microsystems, In Press,(https://doi.org/10.1016/j.micpro.2020.103352) 